\title{
Alteration of the chemoresistant gene expression during chemotherapy for colon cancer: A molecular case report
}

\author{
YUJI TOIYAMA, YASUHIRO INOUE, JUNICHIRO HIRO, EIKI OJIMA, \\ HIDEKI WATANABE, CHIKAO MIKI and MASATO KUSUNOKI \\ Department of Gastrointestinal and Pediatric Surgery, Division of Reparative Medicine, \\ Institute of Life Sciences, Mie University Graduate School of Medicine, Mie, Japan
}

Received August 27, 2007; Accepted December 7, 2007

\begin{abstract}
Chemotherapy remains the main choice of treatment for the management of unresectable or metastatic colorectal cancer. However, drug resistance is a major problem, limiting the effectiveness of the chemotherapies presently used to treat cancer. During treatment, drug resistance can also be acquired by tumors that are initially sensitive to chemotherapy. We present a case of metachronous splenic recurrence after a curative resection of appendical cancer with ovarian metastasis, although the patient had been treated with 5-FU/LV followed by mFOLFOX6 after surgery. Molecular analyses by RT-PCR also showed that the residual tumor after chemotherapy has cancer cells overexpressing 5-FU/1-OHP based chemotherapyresistant genes. Therefore, it was suggested that a careful assessment of the disease status be undertaken during chemotherapy to ensure that the possibility of surgical resection, especially of the re-growth or partial response tumors, was not missed, since several genes, chemoresistant to the agents used, can be induced in residual tumors during chemotherapy.
\end{abstract}

\section{Introduction}

Although surgery is the treatment mainstay for colorectal cancer (CRC), the use of chemotherapy has been considerably expanded because of the development of several new drugs. Chemotherapy remains the choice of treatment for the management of unresectable and metastatic colorectal cancers. In western countries, modern chemotherapy regimens created by feedback from clinical trials have significantly increased the response and survival among such patients. The median overall survival has increased from about 1 year in patients receiving bolus or continuous infusion 5-FU-based treatment

Correspondence to: Dr Masato Kusunoki, Department of Gastrointestinal and Pediatric Surgery, Division of Reparative Medicine, Institute of Life Sciences, Mie University Graduate School of Medicine, 2-174 Edobashi Tsu, Mie 514-8507, Japan

E-mail: kusunoki@clin.medic.mie-u.ac.jp

Key words: chemoresistant gene, chemotherapy, colon cancer, splenic metastasis
(1), to between 14.8 and 20.1 months in patients receiving combinations of 5-FU and irinotecan (CPT-11) (2) or oxaliplatin (L-OHP) (3) as first-line treatments for metastatic disease. However, despite these improvements in survival, the prognosis for patients with metastatic disease still remains poor. Almost all of these patients eventually die from chemoresistant disease $(4,5)$. Therefore, modern chemotherapy aims to prolong survival and deliver a better quality of life (QOL). Nevertheless, there is evidence that some patients initially treated with palliative intent undergo surgical resection of their metastases after chemotherapy, resulting in better survival than that of treatment by chemotherapy alone. However, despite some resounding clinical successes, the significance of multidrug regimens and secondary cytoreduction by metastatic CRC treatment still remains to be fully detailed.

Drug resistance is a major problem which limits the effectiveness of chemotherapies presently used to treat cancer. Certain tumors are intrinsically resistant to chemotherapy prior to treatment. However, drug resistance can also be acquired during treatment by tumors that are initially sensitive to chemotherapy. Drug resistance, whether intrinsic or acquired, is believed to cause treatment failure in $>90 \%$ of patients with metastatic cancer. Obviously, if drug resistance were overcome, the impact on survival would be highly significant (6).

We present a case of metachronous splenic recurrence after a curative resection of appendical cancer with ovarian metastasis, although the patient was treated with 5-FU/LV and 5-FU/LV/1-OHP after surgery. We also report about whether chemoresistant gene expressions alternate under chemotherapy in comparison with a primary and secondary resection sample and discuss the significance of multidrug regimens and secondary cytoreduction in metastatic CRC treatment.

\section{Case Report}

In December 2004, a 70-year old woman was diagnosed with appendical cancer with bil. ovarian metastases. She underwent a right hemicolectomy with bilateral oophorectomy. Histopathological findings in the resected specimen showed mucinous adenocarcinoma with no lymph node metastasis. The tumor was clinically staged as Dukes D (T3NOM1) and the patient received adjuvant chemotherapy. Since first-line chemotherapy for curative colorectal cancer involves modified 


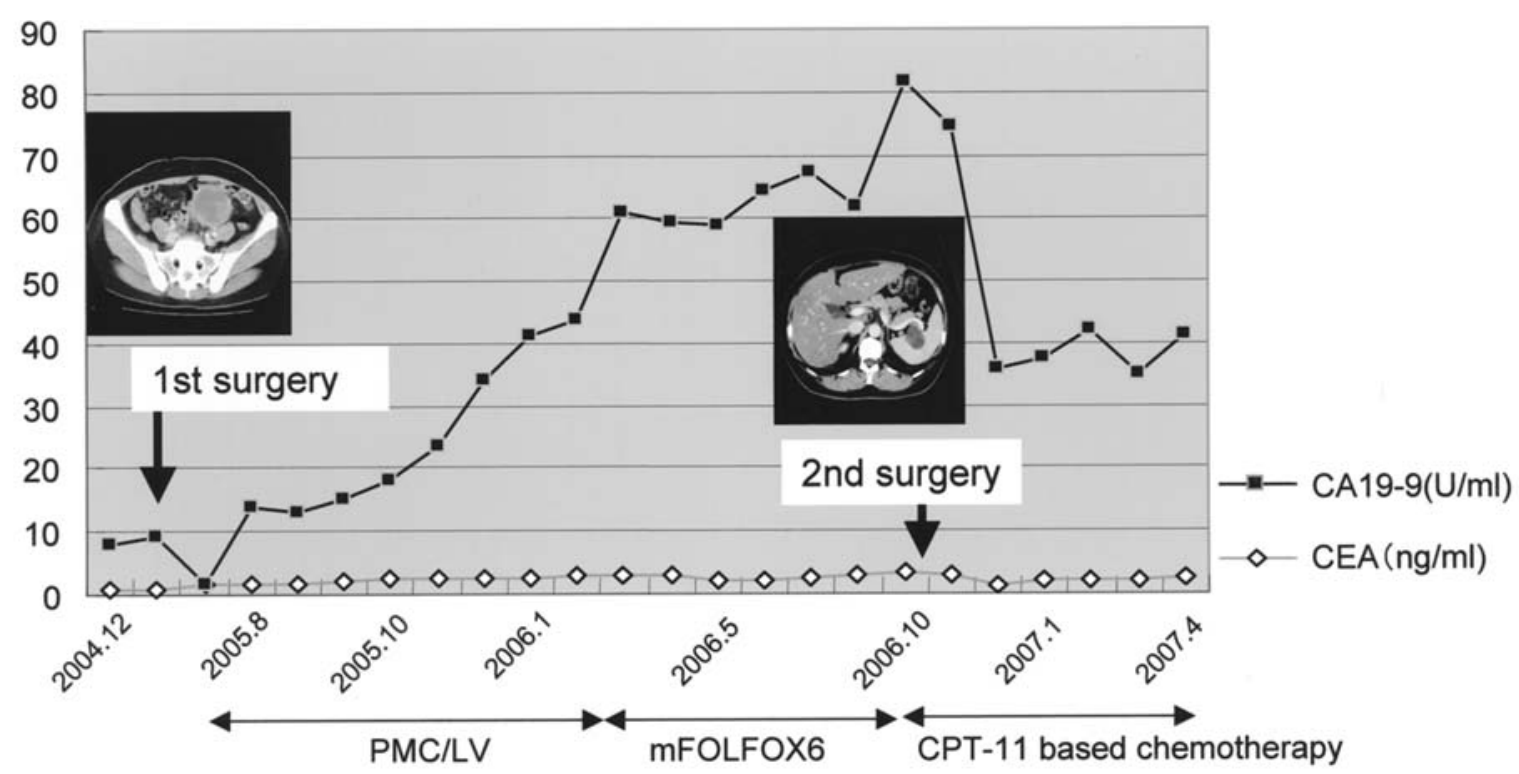

Figure 1. Clinical course and changes in the patient's serum CEA and CA19-9 levels.
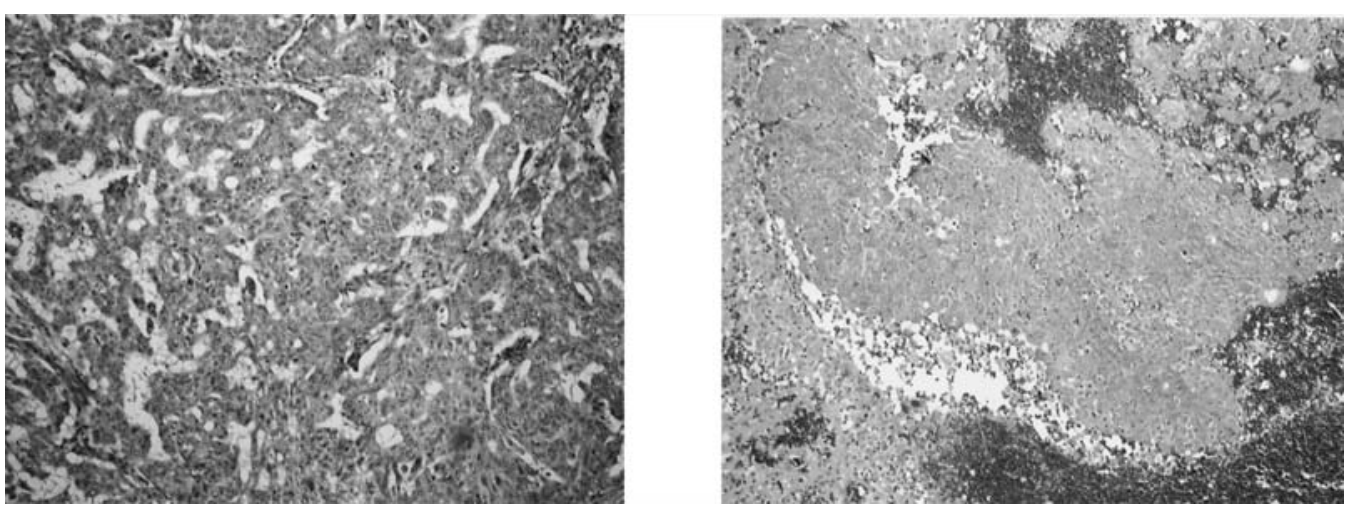

Figure 2. Histological section of the primary colonic tumor located at the appendix with a glandular pattern; x100, hematoxylin and eosin (a). Histological section of the splenic tumor showing a glandular pattern consistent with metastasis from a colonic adenocarcinoma; x100, hematoxylin and eosin (b).

pharmacokinetic modulating chemotherapy (PMC) (7) using Leucovorin $(\mathrm{LV})$, a modified treatment regimen of PMC was administered to the patient. PMC here consisted of continuous intravenous infusion of 5-FU for $24 \mathrm{~h} /$ week and oral administration of UFT (Taiho Pharmaceutical Co., Tokyo, Japan) twice a day for 5-7 days/week (5-FU, $600 \mathrm{mg} / \mathrm{m}^{2} / 24 \mathrm{~h}$ and UFT, $400 \mathrm{mg}$ /day for a week).

The postoperative course was uneventful. During a routine post-surgery checkup, serum tumor markers such as the carcinoembryonic antigen (CEA) and CA19-9, which were initially normalized by surgery, were found to have increased immediately. Although the patient had no symptoms and the computed tomography (CT) detected no recurrence, CA19-9 increased progressively. We changed the chemotherapy treatment regime to mFOLFOX6 (Fig. 1). Increases in the serum CA19-9 level stopped after starting treatment with a combination of 5-FU and 1-OHP for 6 months. However, the CA19-9 level began to rise again in this asymptomatic case, necessitating further imaging studies including a contrast- enhanced abdominal and thoracic $\mathrm{CT}$ which showed an unenhanced mass in the hilum of the spleen, while the liver, lung and the paraaortic area were found to be disease-free. We diagnosed isolated metachronous splenic metastasis from the appendical cancer. The patient underwent splenectomy 17 months after her previous colon cancer surgery. At laparotomy, the tumor was seen in the hilum of the spleen without any capsule invasion macroscopically. There were neither liver nor other intra-abdominal organ metastases. Also, any lymph node involvements at the splenic hilus or paraaortic sites were not detected during the operation. A histological review, including the original tumor, showed that the splenic nodule was consistent with a metastatic adenocarcinoma similar to the original adenocarcinoma of the appendix (Fig. 2).

The postoperative course was uneventful and the patient was discharged on the tenth postoperative day. We instituted a chemotherapy regimen with CPT-11-based chemotherapy at an outpatient clinic. The patient remains with no recurrence at 30 months after the initial treatment. 
Table I. Primer sets used for reverse transcription-polymerase chain reaction.

Gene Primer

\section{TS}

$\begin{array}{ll}\text { Sense } & \text { 5'-GCCTCGGTGTGCCTTTCA-3' } \\ \text { Antisense } & \text { 5'-CCCGTGATGTGCGCAAT-3' }\end{array}$

DPD

Sense 5'-AGGACGCAAGGAGGGTTTG-3'

Antisense 5'-GTCCGCCGAGTCCTTACTGA-3'

TP

Sense

\section{5'-CCTGCGGACGGAATCCT-3'}

Antisense

5'-TCCACGAGTTTCTTACTGAGAATGG-3'

OPRT

Sense

Antisense

5'-CCAGGAGTTCAGTTGGAAGC-3'

ERCC1

Sense

Antisense

5'-GGGAATTTGGCGACGTAATTC-3'

Top I

Sense

Antisense

$$
\begin{aligned}
& \text { 5'-ACAACGATTCCCAGATCGAA-3' } \\
& \text { 5'-CGGTGTTCTCGATCTTTGTG-3' }
\end{aligned}
$$

CE2

Sense

Antisense

B-actin

Sense

Antisense

\section{5'-ACAGAGCCTCGCCTTTGC-3'}

5'-GCGGCGATATCATCATCC-3'

\section{Material and methods}

Patient samples. Surgical specimens of the primary appendical cancer and splenic metastasis were harvested under sterile conditions. Specimens were immediately placed in liquid nitrogen and stored at $-80^{\circ} \mathrm{C}$ until use. A histopathological examination was done on $10 \%$ formalin-fixed, paraffinembedded specimens by a pathologist in our Pathology Division. Use of the patient material was conducted according to the Institutional Review Board guidelines and protocols.

Reverse transcription-PCR analysis. Total RNA from the primary colon cancer and ovarian metastasis was extracted using an RNeasy mid kit (Qiagen Inc., Chatsworth, CA) according to the manufacturer's instructions. Reverse transcription-polymerase chain reaction (RT-PCR) was performed using the specific primers set out in Table I. Optimum cycling parameters in the linear phase of amplification consisted of 23-28 cycles of $30 \mathrm{sec}$ of denaturation at $94^{\circ} \mathrm{C}, 30 \mathrm{sec}$ of annealing at $60^{\circ} \mathrm{C}$ and $1 \mathrm{~min}$ of elongation at $72^{\circ} \mathrm{C}$ for selected genes. A control PCR ( 25 cycles) was also performed with $\beta$-actin as a standard for sample normalization. Amplified products were separated electrophoretically, visualized and photographed under UV light after ethidium bromide staining and then quantified by a CA Analyzer version 2.0 (ATTO, Tokyo, Japan).

\section{Results}

Semi-quantitative reverse transcription-PCR analysis was used to analyze the expression levels of a number of genes indicated in determining sensitivity to 5-FU, 1-OHP and CPT11-based chemotherapy.

We observed that the levels of the 5-FU-anabolizing enzyme thymidine phosphorylase (TP) remained unchanged in the primary appendical cancer and the secondary splenic
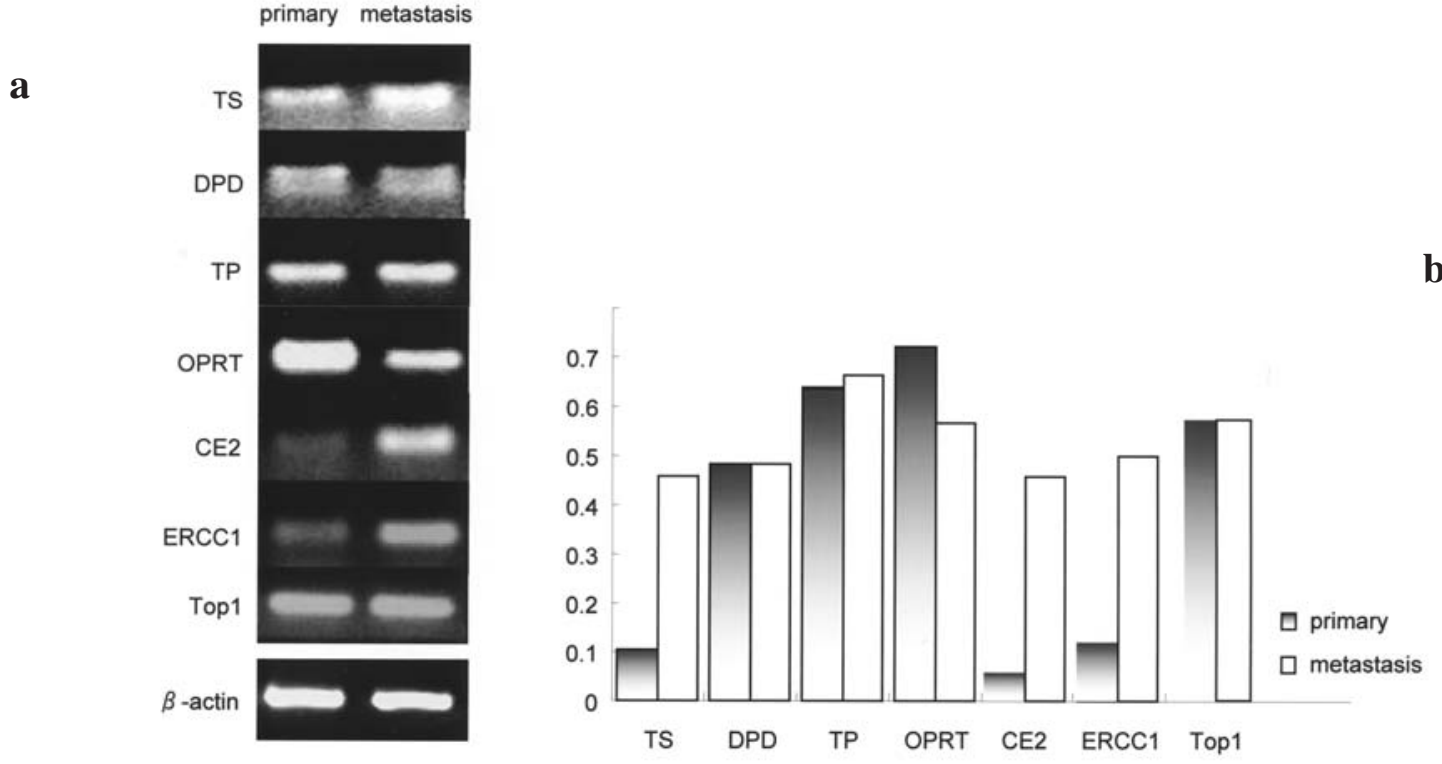

Figure 3. (a) Expression patterns of drug sensitivity genes in the primary and metastasis lesions; thymidylate synthase (TS), dihydropyrimidine dehydrogenase (DPD) gene expression patterns, thymidine phosphorylase (TP), orotate phosphoribosyl transfenase (OPRT), carboxylesterase (CE), topoisomerase I (Top I), and excision repair cross-complementation group1 (ERCC1). (b) The semi-quantification of each gene expression (each gene/ß-actin) was performed using a CA Analyzer version 2.0. 
metastasis that was resistant to 5-FU-based chemotherapy. We also noted that the mRNA levels of the 5-FU-catabolizing enzyme dihydropyrimidine dehydrogenase (DPD) were comparable in the primary and secondary lesions. Notably, the orotate phosphoribosyl transferase (OPRT) expression was lower in the splenic recurrence with the 5-FU-based chemoresistance. Furthermore, the 5-FU target enzyme thymidylate synthase (TS) was overexpressed in the splenic metastasis compared to the primary site.

Then, in the 5-FU/L-OHP resistant splenic metastasis, we found significant increases in the mRNA levels of excision repair cross-complementation group 1 (ERCC1) compared with the primary appendical cancer.

In contrast, the carboxylesterase 2 (CE2) mRNA expression was higher at the site of the recurrence than the primary site, whereas, the expression of topoisomerase I (Top I ) mRNA, a target of CPT-11 was not different between the two lesions (Fig. 3).

\section{Discussion}

Tumors are heterogeneous in humans, with several molecular abnormalities leading to tumor progression and different responses to therapy even in tumors of the same histotype $(8,9)$. This heterogeneity may partly explain the success of multitarget therapeutic strategies in combination chemotherapies. However, during treatment, drug resistance can be acquired by tumors that are initially sensitive to chemotherapy. It is possible that the ability to predict residual tumor responses to cytotoxic drugs is as important as predicting primary tumor responses.

In the case reported here, we showed a metachronous splenic metastasis from appendical cancer during 5-FU/LV treatment, which progressed the disease despite changing to 5-FU/LV/1-OHP. Therefore, we investigated how chemoresistant genes in the metastasis lesion modified during chemotherapy as compared to the primary appendical cancer.

The mechanisms involved in the resistance to chemotherapy usually involve the up-regulation of resistance mechanisms or down-regulation of the target and its related genes. Examples of the former include repairing DNA damage such as ERCC1 known as 1-OHP resistant factor $(16,17)$, while the latter include Top I, targets of CPT-11 and CE2, the active metabolite to the target $(19,20)$. As for $5-\mathrm{FU}$, the target is TS and the representative rate limiting enzymes are DPD, TP and OPRT $(10,11,14,15)$. The present study aimed to clinically evaluate the role of multidrug administration and cytoreductive surgery during therapeutic chemotherapy for colon cancer. To best clarify the clinical significance, we focused on the chemoresistant mechanism.

We found no modulation of the DPD or TP mRNA expression between primary and secondary lesions. However, up-regulated TS mRNA and down-regulated OPRT mRNA were confirmed in the 5-FU-resistant metastatic lesion. This is consistent with several in vitro studies that have demonstrated correlations between TS or OPRT levels and 5-FU drug sensitivity $(12,13)$. There are relatively few predictive biomarkers currently available to identify patients most likely to respond to 1-OHP. However, one important factor is ERCC1, which allows for the repair of DNA damage. Our case also demonstrated an elevated ERCC1 and TS mRNA expression in the splenic metastasis lesion that was resistant to the 5-FU/1-OHP regimen, according to earlier reports (18).

These results suggested that the residual tumor after chemotherapy had 5-FU/1-OHP-based chemotherapy-resistant components and the same chemotherapy on its own would not provide any survival benefit. Therefore, secondary surgery is one possible option to eradicate the residual chemoresistant component of the malignancy. Notably, though the expression of Top I mRNA was not different, CE2 mRNA expression was higher at the recurrent site than the primary lesion. This may suggest that CPT-11-based chemotherapy was useful in an adjuvant chemotherapy setting after splenectomy in our case.

In conclusion, this molecular level case report indicated that treatment with anti-tumor agents can induce several genes that are chemoresistant to drugs used during chemotherapy. This demonstrated that it is important to investigate the ability to predict a chemoresponse to the primary lesion but also to a recurrent lesion. Since few studies have thus far investigated molecular profiles in residual tumors during chemotherapy, we need to change chemotherapy regimens and assess the possibility of surgical resection to metastatic sites as chemoresistant tumor cells are emerging. We believe that the individualization of therapy such as cytoreduction or chemotherapy along with the molecular phenotype of residual tumors during chemotherapy will improve survival.

\section{References}

1. Meta-analysis group in cancer. Efficacy of intravenous continuous infusion of fluorouracil compared with bolus administration in advanced colorectal cancer. J Clin Oncol 16: 301-308, 1998.

2. Saltz LB, Cox JV, Blanke C, et al: Irinotecan plus fluorouracil and leucovorin for metastatic colorectal cancer. Irinotecan study group. N Engl J Med 343: 905-914, 2000.

3. de Gramont A, Figer A, Seymour M, et al: Leucovorin and fluorouracil with or without oxaliplatin as first-line treatment in advanced colorectal cancer. J Clin Oncol 18: 2938-2947, 2000.

4. Wagner JS, Adson MA and Van Heerden JA: The natural history of hepatic metastases from colorectal cancer: A comparison with resective treatment. Ann Surg 199: 502-508, 1984.

5. Fong Y: Surgical therapy of hepatic colorectal metastasis. CA Cancer J Clin 49: 231-235, 1999.

6. Longley DB and Johnston PG: Molecular mechanisms of drug resistance. J Pathol 205: 275-292, 2005.

7. Kusunoki M, Yanagi H, Noda $M$ and Yamamura T: The usefulness of pharmacokinetic modulating chemotherapy (UFT plus $5 \mathrm{FU}$ ) in the treatment of unresectable colorectal carcinomas. Oncol Rep 6: 547-552, 1998.

8. Liggett SB: Pharmacogenetic applications of the human genome project. Nat Med 7: 281-283, 2001.

9. Takayama T, Miyanishi K, Hayashi T, Sato Y and Niitsu Y: Colorectal cancer: Genetics of development and metastasis. J Gastroenterol 41: 185-192, 2006.

10. Salonga D, Danenberg KD, Johnson M, et al: Colorectal tumors responding to 5-fluorouracil have low gene expression levels of dihydropyrimidine dehydrogenase, thymidylate synthase and thymidine phosphorylase. Clin Cancer Res 6: 1322-1327, 2000.

11. Takebe N, Zhao SC, Ural AU, et al: Retroviral transduction of human dihydropyrimidine dehydrogenase cDNA confers resistance to 5-fluorouracil in murine hematopoietic progenitor cells and human CD34-enriched peripheral blood progenitor cells. Cancer Gene Ther 8: 966-973, 2001

12. Inaba M, Mitsuhashi J, Sawada H, et al: Reduced activity of anabolizing enzymes in 5-fluorouracil-resistant human stomach cancer cells. Jpn J Cancer Res 87: 212-220, 1996. 
13. Chung YM, Park S, Park JK, Kim Y, Kang Y and Yoo YD: Establishment and characterization of 5-fluorouracil-resistant gastric cancer cells. Cancer Lett 159: 95-101, 2000.

14. Fujii R, Seshimo A and Kameoka S: Relationships between the expression of thymidylate synthase, dihydropyrimidine dehydrogenase, and orotate phosphoribosyl transferase and cell proliferative activity and 5-fluorouracil sensitivity in colorectal carcinoma. Int J Clin Oncol 8: 72-78, 2003.

15. Isshi K, Sakuyama T, Gen T, et al: Predicting 5-FU sensitivity using human colorectal cancer specimens: comparison of tumor dihydropyrimidine dehydrogenase and orotate phosphoribosyl transferase activities with in vitro chemosensitivity to 5-FU. Int J Clin Oncol 7: 335-342, 2002.

16. Hector S, Bolanowska-Higdon W, Zdanowicz J, Hitt S and Pendyala L: In vitro studies on the mechanisms of oxaliplatin resistance. Cancer Chemother Pharmacol 48: 398-406, 2001.
17. Arnould S, Hennebelle I, Canal P, Bugat R and Guichard S: Cellular determinants of oxaliplatin sensitivity in colon cancer cell lines. Eur J Cancer 39: 112-119, 2003.

18. Shirota Y, Stoehlmacher J, Brabender J, et al: ERCC1 and thymidylate synthase mRNA levels predict survival for colorectal cancer patients receiving combination oxaliplatin and fluorouracil chemotherapy. J Clin Oncol 19: 4298-4304, 2001.

19. Humerickhouse R, Lohrbach K, Li L, Bosron WF and Dolan ME: Characterization of CPT-11 hydrolysis by human liver carboxylesterase isoforms hCE-1 and hCE-2. Cancer Res 1: $1189-1192,2000$

20. Xu G, Zhang W, Ma MK and McLeod HL: Human carboxylesterase 2 is commonly expressed in tumor tissue and is correlated with activation of irinotecan. Clin Cancer Res 8: 2605-2611, 2002. 